\title{
Upper Bound of Primitive Exponents of a Class of Two-colored Digraph with $n$ Vertices
}

\author{
Meijin Luo ${ }^{1, a}$ and $\mathrm{Xi} \mathrm{Li}{ }^{2, b}$ \\ ${ }^{1}$ School of Mathematics and Statistics, Hechi University Yizhou, Guangxi 546300, P.R. China \\ ${ }^{2}$ Department of Basic Education, Shanxi Yuncheng Vocational College of Agriculture, Yuncheng, \\ Shanxi 046012,P.R. China. \\ ameijin322@163.com, ${ }^{\mathrm{b}} 924676854 @ q q . c o m$
}

\section{Keywords: Two-colored digraph; Exponent; Upper bound}

\begin{abstract}
A two-colored directed digraph $\mathscr{D}$ is primitive if and only if there exist nonnegative integers $h$ and $k$ with $h+k>0$ such that for each pair $(i, j)$ of vertices there is a $(h, \ell$-walk in $\mathscr{D}$ from $i$ to $j$. A $(h, k)$ -walk from $i$ to $j$ consisting of $h$ red arcs and $k$ blue arcs. The exponent of the primitive two-colored digraph $\mathscr{D}$, denoted $\exp (\mathcal{D})$, is defined to be the smallest value of $\hbar+\kappa$ over all such $\hbar$ and $k_{\text {. A class of }}$ two-colored digraphs with two cycles whose uncolored digraph has $n$ vertices and consists of one $n$ -cycle and one 3 -cycle is considered. The upper bound of primitive exponent and characteristic of extremal two-colored digraphs are given.
\end{abstract}

\section{Introduction}

Let $D$ be a digraph. A walk in $D$ of length $l$ is a sequence $v_{1}, v_{2}, \cdots, v_{l+1}$ of vertices such that there is an arc in $D$ from $v_{i}$ to $v_{i+1}$ for $i=1,2, \cdots, l$. The walk is a path if the vertices $v_{1}, v_{2}, \cdots, v_{l+1}$ are distinct [1]. A two-colored digraph is a digraph whose arcs are colored red and blue. We allow loops and both a red arc and blue arc from $i$ to $j$ for all pairs $(i, j)$ of vertices. $D$ is strongly connected provided for each pair $(i, j)$ of vertices there is a walk in $D$ from $i$ to $j$. Given a walk $\omega$ in $D, r(\omega)$ (respectively, $b(\omega)$ ) is the number of red arcs (respectively, blue arcs) of $\omega$, and the composition of $\omega$ is the vector $(r(\omega), b(\omega))$ or $(r(\omega), b(\omega))^{T}$.

A two-colored directed digraph $D$ is primitive if and only if there exist nonnegative integers $h$ and $k$ with $h+k>0$ such that for each pair $(i, j)$ of vertices there is a $(h, k)$-walk in $D$ from $i$ to $j$. A $(h, k)$-walk from $i$ to $j$ consisting of $h$ red arcs and $k$ blue arcs. The exponent of the primitive two-colored digraph $D$, denoted $\exp (D)$, is defined to be the smallest value of $h+k$ over all such $h$ and $k$.

Let $C=\left\{\gamma_{1}, \gamma_{2}, \cdots, \gamma_{l}\right\}$ be the set of cycles of $D$. Set $M$ to be the $2 \times l$ matrix whose $i$ th column is the composition of $\gamma_{i}$. We call $M$ the cycle matrix of $D$. The content of $M$, denoted content $(M)$, is defined to be 0 if the rank of $M$ is less than 2 and the greatest common divisor of all $2 \times 2$ minors of $M$, otherwise.

Lemma 1.1 Let $D$ be a two-colored digraph having at least one red $\operatorname{arc}$ and one blue arc. Then $D$ is primitive if and only if $D$ is strongly connected and content $(M)=1[2]$.

It is well known that there is a natural correspondence between two-colored digraphs and nonnegative matrix pairs ([2]). The concept of exponent of nonnegative matrix pair arises in the study of finite Markov chains([2,3]), and some results have already been obtained ([3-10]).In this paper, we consider the two-colored digraph $D$ in Figure. 1 with $n$ vertices which has at least one red and one blue arc.

For $n \geq 4$, the greatest common factor of $n$ and 3 to be 1,we consider the two-colored digraph that has at least one red arc and one blue arc, and whose uncolored digraph is that in Fig. 1. 


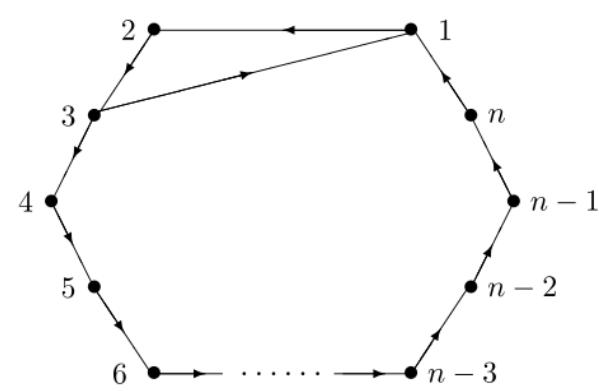

Figure 1. Finite Uncolored digraph of D

Clearly, $D$ has only two cycles that one is an $n$-cycle and the other one is an 3 -cycle. If all of arcs on 3 -cycle are red or blue, then content $(M) \neq 1, D$ is not primitive. Without loss of generality, we may assume that the cycle matrix of $D$ is

$$
M=\left[\begin{array}{cc}
a & 1 \\
n-a & 2
\end{array}\right]
$$

or

$$
M=\left[\begin{array}{cc}
a & 2 \\
n-a & 1
\end{array}\right]
$$

for some nonnegative integer $a$.

Theorem 1.2 If the cycle matrix of $D$ is (1), $D$ is primitive if and only if $a=\frac{n \pm 1}{3}$.

Proof From $(1), \operatorname{det}(M)=2 a-(n-a)$. By Lemma 1.1, $D$ is primitive if and only if $\operatorname{content}(M)=1$, that is, $\operatorname{det}(M)= \pm 1$. Then the theorem follows.

Theorem 1.3 If the cycle matrix of $D$ is (2), $D$ is primitive if and only if $a=\frac{2 n \pm 1}{3}$.

Proof From (2), $\operatorname{det}(M)=a-2(n-a)$. By Lemma 1.1, $D$ is primitive if and only if $\operatorname{content}(M)=1$, that is, $\operatorname{det}(M)= \pm 1$. Then the theorem follows.

\section{The Upper Bound of the Exponents}

In this section, we will give the upper bound on the primitive exponent for $D$. By Theorem 1.2, we obtain the 3 -cycle of $D$ contains exactly one red arc and two blue arcs. By Theorem 1.3, we obtain the 3 -cycle of $D$ contains exactly two red arcs and one blue arc.

Theorem 2.1 If the cycle matrix of $D$ is (1), $D$ is primitive and $a=\frac{n+1}{3}$, then $\exp (D) \leq \frac{4 n^{2}+3 n-1}{3}$

Proof Form Theorem 1.2, we know the inverse matrix of $M$ is $\left[\begin{array}{cc}2 & -1 \\ -\frac{2 n-1}{3} & \frac{n+1}{3}\end{array}\right]$. We only prove that between each pair $(i, j)$ of vertices of $D$ there is an $\left(\frac{4 n^{2}+5 n+1}{9}, \frac{8 n^{2}+4 n-4}{9}\right)$-walk. For any pair $(i, j)$ vertices of $D$, let $P_{i j}$ be the shortest path in $D$ from $i$ to $j$, and denote $r\left(P_{i j}\right)=s$ and $b\left(P_{i j}\right)=t$. We see that

$$
\left[\begin{array}{l}
s \\
t
\end{array}\right]+\left(\frac{2 n+2}{3}-2 s+t\right)\left[\begin{array}{c}
\frac{n+1}{3} \\
\frac{2 n-1}{3}
\end{array}\right]+\left(\frac{(2 n-1)(n+1)}{9}+\frac{2 n-1}{3} s-\frac{n+1}{3} t\right)\left[\begin{array}{l}
1 \\
2
\end{array}\right]=\left[\begin{array}{c}
\frac{4 n^{2}+5 n+1}{9} \\
\frac{8 n^{2}+4 n-4}{9}
\end{array}\right] .
$$


Noting that $0 \leq s \leq \frac{n+1}{3}, 0 \leq t \leq \frac{2 n-1}{3}$, it is easy to see that $\rho_{1} \geq 0$ and $\rho_{2} \geq 0$. If $s=\frac{n+1}{3}$, then $t \geq 0$ and if $t=\frac{2 n-1}{3}$, then $s \geq 0$. This gives

$$
\exp (D) \leq \frac{4 n^{2}+5 n+1}{9}+\frac{8 n^{2}+4 n-4}{9}=\frac{4 n^{2}+3 n-1}{3} .
$$

The theorem follows.

Form Theorem 2.1, the following results are clear.

Theorem 2.2 If the cycle matrix of $D$ is (1), $D$ is primitive and $a=\frac{n-1}{3}$, then $\exp (D) \leq \frac{4 n^{2}-1}{3}$.

Theorem 2.3 If the cycle matrix of $D$ is (2), $D$ is primitive and $a=\frac{2 n+1}{3}$, then $\exp (D) \leq \frac{4 n^{2}-1}{3}$.

Theorem 2.4 If the cycle matrix of $D$ is (2), $D$ is primitive and $a=\frac{2 n-1}{3}$, then $\exp (D) \leq \frac{4 n^{2}+3 n-1}{3}$

\section{The Extremal Two-Colored Digraphs}

In this section, we determine the two-colored digraphs that achieve the upper bound of primitive exponents of $D$.

Lemma 3.1 If the cycle matrix of $D$ is (1), $D$ is primitive and $a=\frac{n+1}{3}$, then $\exp (D)=\frac{4 n^{2}+3 n-1}{3}$ if and only if the $\frac{n+1}{3}$ red arcs are consecutive on the $n$-cycle, that is, the $\frac{2 n-1}{3}$ blue arcs are consecutive on the $n$-cycle.

Proof Sufficiency: Form Theorem 2.1, we only proof $\exp (D) \geq \frac{4 n^{2}+3 n-1}{3}$.

Suppose that $(h, k)$ is a pair of nonnegative integers such that for all pairs $(i, j)$ of vertices there is an $(h, k)$-walk from $i$ to $j$. By considering $i=j=n$, we see that there exist nonnegative integers $u$ and $v$ with

$$
\left[\begin{array}{l}
h \\
k
\end{array}\right] M=\left[\begin{array}{l}
u \\
v
\end{array}\right] .
$$

Taking $i$ and $j$ to be the initial vertex and the terminal vertex of the $\frac{n+1}{3}$ red arcs are consecutive on the $n$-cycle, and this path has composition $\left(\frac{n+1}{3}, 0\right)$. Hence

$$
M z=\left[\begin{array}{c}
h-\frac{n+1}{3} \\
k
\end{array}\right]
$$

has a nonnegative integer solution. Necessarily,

$$
z=M^{-1}\left[\begin{array}{c}
h-\frac{n+1}{3} \\
k
\end{array}\right]=\left[\begin{array}{l}
u \\
v
\end{array}\right]-M^{-1}\left[\begin{array}{c}
\frac{n+1}{3} \\
0
\end{array}\right]=\left[\begin{array}{l}
u \\
v
\end{array}\right]-\left[\begin{array}{c}
\frac{2 n+2}{3} \\
-\frac{(n+1)(2 n-1)}{9}
\end{array}\right] \geq 0
$$

So $u \geq \frac{2 n+2}{3}$. Next take $i$ and $j$ to be the terminal vertex and the initial vertex of the $\frac{n+1}{3}$ red arcs are consecutive on the $n$-cycle, and this path has composition $\left(0, \frac{2 n-1}{3}\right)$. Hence 


$$
M Z=\left[\begin{array}{c}
h \\
k-\frac{2 n-1}{3}
\end{array}\right]
$$

has a nonnegative integer solution. Necessarily,

$$
Z=M^{-1}\left[\begin{array}{c}
h \\
k-\frac{2 n-1}{3}
\end{array}\right]=\left[\begin{array}{l}
u \\
v
\end{array}\right]-M^{-1}\left[\begin{array}{c}
0 \\
\frac{2 n-1}{3}
\end{array}\right]=\left[\begin{array}{l}
u \\
v
\end{array}\right]-\left[\begin{array}{c}
-\frac{2 n-1}{3} \\
\frac{(2 n-1)(n+1)}{9}
\end{array}\right] \geq 0 .
$$

So $v \geq \frac{(2 n-1)(n+1)}{9}$. Thus

$$
h+k=\left[\begin{array}{ll}
1 & 1
\end{array}\right] M\left[\begin{array}{l}
u \\
v
\end{array}\right] \geq\left[\begin{array}{ll}
n & 3
\end{array}\right]\left[\begin{array}{c}
\frac{2 n+2}{3} \\
\frac{(2 n-1)(n+1)}{9}
\end{array}\right]=\frac{4 n^{2}+3 n-1}{3} .
$$

Then $\exp (D) \geq \frac{4 n^{2}+3 n-1}{3}$.

Necessity: We only need to prove that the $\frac{n+1}{3}$ red arcs are not consecutive on the $n$-cycle; or the $\frac{2 n-1}{3}$ blue arcs are not consecutive on the $n-$ cycle, then $\exp (D)<\frac{4 n^{2}+3 n-1}{3}$.

For any pair $(i, j)$ of vertices of $D$, let $P_{i j}$ be the shortest path in $D$ from $i$ to $j$, and denote $r\left(P_{i j}\right)=s$ and $b\left(P_{i j}\right)=t$. We consider the walk that starts at vertex $i$, follows $P_{i j}$ to vertex $j$, goes $\rho_{1}$ times around the $n$-cycle, and $\rho_{2}$ times around the 3 -cycle. Taking $\rho_{1}=\frac{2 n-1}{3}-2 s+t$ and $\rho_{2}=$ $\frac{(2 n-1)(n-2)}{9}+\frac{2 n-1}{3} s-\frac{n+1}{3} t$. We see that

$$
\left[\begin{array}{l}
s \\
t
\end{array}\right]+\rho_{1}\left[\begin{array}{c}
\frac{n+1}{3} \\
\frac{2 n-1}{3}
\end{array}\right]+\rho_{2}\left[\begin{array}{l}
1 \\
2
\end{array}\right]=\left[\begin{array}{c}
\frac{4 n^{2}-4 n+1}{9} \\
\frac{8 n^{2}-14 n+5}{9}
\end{array}\right] .
$$

Noting that $0 \leq s \leq \frac{n+1}{3}, 0 \leq t \leq \frac{2 n-1}{3}$, it is easy to see that $\rho_{1} \geq 0$ and $\rho_{2} \geq 0$. If $s=\frac{n+1}{3}$, then $t \geq 1$ and if $t=\frac{2 n-1}{3}$, then $s \geq 1$. This gives

$$
\exp (D) \leq \frac{4 n^{2}-4 n+1}{9}+\frac{8 n^{2}-14 n+5}{9}=\frac{4 n^{2}-6 n+2}{3}<\frac{4 n^{2}+3 n-1}{3} .
$$

Combining Theorem2.1, the theorem follows.

As in the proof of Lemma 3.1, we can prove the following results.

Lemma 3.2 If the cycle matrix of $D$ is (1), $D$ is primitive and $a=\frac{n-1}{3}$, then $\exp (D)=\frac{4 n^{2}-1}{3}$ if and only if the $\frac{n-1}{3}$ red arcs are consecutive on the $n$-cycle, that is, the $\frac{2 n+1}{3}$ blue arcs are consecutive on the $n$-cycle.

Lemma 3.3 If the cycle matrix of $D$ is (2), $D$ is primitive and $a=\frac{2 n+1}{3}$, then $\exp (D)=\frac{4 n^{2}-1}{3}$ if and only if the $\frac{2 n+1}{3}$ red arcs are consecutive on the $n$-cycle, that is, the $\frac{n-1}{3}$ blue arcs are 
consecutive on the $n$-cycle.

Lemma 3.4 If the cycle matrix of $D$ is (2), $D$ is primitive and $a=\frac{2 n-1}{3}$, then $\exp (D)=\frac{4 n^{2}+3 n-1}{3}$ if and only if the $\frac{2 n-1}{3}$ red arcs are consecutive on the $n$-cycle, that is, the $\frac{n+1}{3}$ blue arcs are consecutive on the $n$-cycle.

\section{Summary}

Form Theorem 1.2-Theorem 1.3 and Lemma 3.1-Lemma 3.4, we can find the upper bound of $D$.

Theorem 4.1 $D$ is primitive, then $\exp (D) \leq \frac{4 n^{2}+3 n-1}{3}$.

\section{Acknowledgements}

The authors would like to thank all referees for their valuable suggestions on earlier versions of this paper. This research was supported by Project of Guangxi Colleges and Universities (NO.YB2014335) and Scientific and Technologial Innovation Programs of Higher Education Institutions in Shanxi(NO.20151113).

\section{References}

[1] R.A. Brualdi, H.J. Ryser, Combinatorial Matrix Theory, Encyclopedia of Mathematics and its Applications, vol. 39, Cambridge University Press, Cambridge, 1991.

[2] B.L. Shader, S. Suwilo, Exponents of nonnegative matrix pairs, Linear Algebra Appl, 363 (2003), pp. 275-293.

[3] A. Berman, R. Plemmons, Nonnegative Matrices in the Mathematical Science, Classics in Applied Mathematics. Vol. 9, SIAM, Philadelphia, PA, 1994.

[4] Y.B. Gao, Y.L. Shao, Exponent of two-colored digraphs with two cycles, Linear Algebra Appl, 407 (2005), pp. 263-276.

[5] Y.B. Gao, Y.L. Shao, Exponent of two-colored double directed cycles, Journal of Natural Science of Heilongjiang University, 4 (2004), pp. 55-58.

[6] Y.L. Shao, Y.B. Gao, L. Sun, Exponent of a class of two-colored digraphs, Linear and Multilinear Algrbra, 53:3 (2005), pp. 175-188.

[7] M.J. Luo, Y.B. Gao, Primitive exponent of three-colored digraphs with three cycles, Journal of Shandong University (Natural Science), 43:1 (2008), pp. 65-72. (In Chinese)

[8] Z.X. Bai, Y.L. Shao, Exponents of a class of two-colored digraphs, Journal of North University of China (Natural Science Edition), 28:2 (2007), pp. 100-103. (In Chinese)

[9] M.J. Luo, Upper bound on primitive exponent of two-colored digraphs, Mathematics in Practice and Theory, 43:23 (2013), pp. 1-9. (In Chinese)

[10] C.F. Liu, Y.B. Gao, The m-competition indices of all primitive digraphs of order $\mathrm{n}$ only with $\left(\begin{array}{ll}n & 1\end{array}\right)$-cycles and ( $n$ 2)-cycles, Journal of Natural Science of Heilongjiang University, 33:1 (2016), pp. 41-49. 